\title{
Third Order Adjoint Sensitivity and Uncertainty Analysis of an OECD/NEA Reactor Physics Benchmark: III. Response Moments
}

\author{
Ruixian Fang, Dan Gabriel Cacuci* \\ Center for Nuclear Science and Energy, Department of Mechanical Engineering, University of South Carolina, Columbia, SC, \\ USA \\ Email: fangr@cec.sc.edu, *cacuci@cec.sc.edu
}

\begin{abstract}
How to cite this paper: Fang, R.X. and Cacuci, D.G. (2020) Third Order Adjoint Sensitivity and Uncertainty Analysis of an OECD/NEA Reactor Physics Benchmark: III. Response Moments. American Journal of Computational Mathematics, 10, 559-570. https://doi.org/10.4236/ajcm.2020.104031
\end{abstract}

Received: October 18, 2020

Accepted: December 6, 2020

Published: December 9, 2020

Copyright $\odot 2020$ by author(s) and Scientific Research Publishing Inc. This work is licensed under the Creative Commons Attribution International License (CC BY 4.0).

http://creativecommons.org/licenses/by/4.0/

(c) (i) Open Access

\begin{abstract}
The $(180)^{3}$ third-order mixed sensitivities of the leakage response of a polyethylene-reflected plutonium (PERP) experimental benchmark with respect to the benchmark's 180 microscopic total cross sections have been computed in accompanying works [1] [2]. This work quantifies the contributions of these $(180)^{3}$ third-order mixed sensitivities to the PERP benchmark's leakage response distribution moments (expected value, variance and skewness) and compares these contributions to those stemming from the corresponding first- and second-order sensitivities of the PERP benchmark's leakage response with respect to the total cross sections. The numerical results obtained in this work reveal that the importance of the $3^{\text {rd }}$-order sensitivities can surpass the importance of the $1^{\text {st }}$ and $2^{\text {nd }}$-order sensitivities when the parameters' uncertainties increase. In particular, for a uniform standard deviation of $10 \%$ of the microscopic total cross sections, the $3^{\text {rd }}$-order sensitivities contribute $80 \%$ to the response variance, whereas the contribution stemming from the $1^{\text {st }}$ - and $2^{\text {nd }}$-order sensitivities amount only to $2 \%$ and $18 \%$, respectively. Consequently, neglecting the $3^{\text {rd }}$-order sensitivities could cause a very large non-conservative error by under-reporting the response variance by a factor of $506 \%$. The results obtained in this work also indicate that the effects of the $3^{\text {rd }}$-order sensitivities are to reduce the response's skewness in parameter space, rendering the distribution of the leakage response more symmetric about its expected value. The results obtained in this work are the first such results ever published in reactor physics. Since correlations among the group-averaged microscopic total cross sections are not available, only the effects of typical standard deviations for these cross sections could be considered. Due to this lack of correlations among the cross sections, the effects of the mixed $3^{\text {rd }}$-order sensitivities
\end{abstract}


could not be quantified exactly at this time. These effects could be quantified only when correlations among the group-averaged microscopic total cross sections would be obtained experimentally by the nuclear physics community.

\section{Keywords}

Polyethylene-Reflected Plutonium Sphere, 3rd-Order Sensitivities, 1st-Order, 2nd-Order and 3rd-Order Uncertainty Analysis, Microscopic Total Cross Sections, Expected Value, Variance and Skewness of Response Distribution

\section{Introduction}

The accompanying works [1] [2] have computed exactly and efficiently the (180) ${ }^{3}$ $=5,832,000$ third-order sensitivities of the leakage response, of the OECD/NEA subcritical polyethylene-reflected plutonium (acronym: PERP) metal fundamental physics benchmark [3], with respect to the benchmark's 180 group-averaged microscopic total cross sections. This work presents the third-order uncertainty analysis of the PERP benchmark's leakage response by using the results obtained in [2]. Specifically, the effects of the $3^{\text {rd }}$-order sensitivities on the PERP benchmark's leakage response distribution moments (e.g., expected values, variance and skewness, etc.) in the space of parameters (microscopic total cross sections) will be quantified and compared with the contributions stemming from the corresponding $1^{\text {st }}$ - and $2^{\text {nd }}$-order sensitivities, underscoring the importance of the $3^{\text {rd }}$-order sensitivities.

This work is organized as follows: Section 2 presents the expressions used for the $3^{\text {rd }}$-order uncertainty analysis of the PERP leakage response, which is derived from the general expressions for third-order uncertainty analysis conceived by Cacuci [4]. Section 3 presents the numerical results for the contributions of the $3^{\text {rd }}$-order sensitivities to the leakage response distribution's first three moments (i.e., expected value, variance and skewness) in the space of parameters (microscopic total cross section). The $3^{\text {rd }}$-order contributions are compared with the corresponding effects stemming from the $1^{\text {st }}$ and $2^{\text {nd }}$-order sensitivities. Section 4 concludes and highlights the important findings obtained in this work, which is the first third-order uncertainty analysis ever performed in reactor physics.

\section{Expressions for Third-Order Uncertainty Analysis of the PERP Leakage Response}

Knowledge of the first-, second-, and third-order sensitivities is required to compute the following moments of the response distribution:

1) Up to $3^{\text {rd }}$-order response sensitivities, the $1^{\text {st }}$-order moment (expected value) of a response $R_{k}$ has the following expression [4]: 


$$
\begin{aligned}
E\left(r_{k}\right)= & r_{k}\left(\boldsymbol{\alpha}^{0}\right)+\frac{1}{2} \sum_{i=1}^{N_{\alpha}} \sum_{j=1}^{N_{\alpha}} \frac{\partial^{2} r_{k}\left(\boldsymbol{\alpha}^{0}\right)}{\partial \alpha_{i} \partial \alpha_{j}} \rho_{i j} \sigma_{i} \sigma_{j} \\
& +\frac{1}{6} \sum_{i=1}^{N_{\alpha}} \sum_{j=1}^{N_{\alpha}} \sum_{k=1}^{N_{\alpha}} \frac{\partial^{3} r_{k}\left(\boldsymbol{\alpha}^{0}\right)}{\partial \alpha_{i} \partial \alpha_{j} \partial \alpha_{k}} t_{i j k} \sigma_{i} \sigma_{j} \sigma_{k}+O\left(\sigma^{4}\right),
\end{aligned}
$$

where $\rho_{i j}$ denotes the correlation coefficient between parameters $\alpha_{i}$ and $\alpha_{j}$, while $\sigma_{i}$ denotes the standard deviation of the model parameter $\alpha_{i}$.

2) Up to $3^{\text {rd }}$-order response sensitivities, the $2^{\text {nd }}$-order moment (covariance) of two responses $\left(R_{k}, R_{\ell}\right)$ has the following expression [4]:

$$
\begin{aligned}
& \operatorname{cov}\left(R_{k}, R_{\ell}\right)=\sum_{i=1}^{N_{\alpha}} \sum_{j=1}^{N_{\alpha}}\left(\frac{\partial R_{k}}{\partial \alpha_{i}} \frac{\partial R_{\ell}}{\partial \alpha_{j}}\right) \rho_{i j} \sigma_{i} \sigma_{j} \\
& +\frac{1}{2} \sum_{i=1}^{N_{\alpha}} \sum_{j=1}^{N_{\alpha}} \sum_{\mu=1}^{N_{\alpha}}\left(\frac{\partial^{2} R_{k}}{\partial \alpha_{i} \partial \alpha_{j}} \frac{\partial R_{\ell}}{\partial \alpha_{\mu}}+\frac{\partial R_{k}}{\partial \alpha_{i}} \frac{\partial^{2} R_{l}}{\partial \alpha_{j} \partial \alpha_{\mu}}\right) t_{i j \mu} \sigma_{i} \sigma_{j} \sigma_{\mu} \\
& +\frac{1}{4} \sum_{i=1}^{N_{\alpha}} \sum_{j=1}^{N_{\alpha}} \sum_{\mu=1}^{N_{\alpha}} \sum_{v=1}^{N_{\alpha}}\left(\frac{\partial^{2} R_{k}}{\partial \alpha_{i} \partial \alpha_{j}}\right)\left(\frac{\partial^{2} R_{\ell}}{\partial \alpha_{\mu} \partial \alpha_{v}}\right)\left(q_{i j \mu \nu}-\rho_{i j} \rho_{\mu v}\right) \sigma_{i} \sigma_{j} \sigma_{\mu} \sigma_{v} \\
& +\frac{1}{6} \sum_{i=1}^{N_{\alpha}} \sum_{j=1}^{N_{\alpha}} \sum_{\mu=1}^{N_{\alpha}} \sum_{v=1}^{N_{\alpha}}\left(\frac{\partial R_{k}}{\partial \alpha_{i}} \frac{\partial^{3} R_{l}}{\partial \alpha_{j} \partial \alpha_{\mu} \partial \alpha_{v}}+\frac{\partial R_{l}}{\partial \alpha_{i}} \frac{\partial^{3} R_{k}}{\partial \alpha_{j} \partial \alpha_{\mu} \partial \alpha_{v}}\right) q_{i j \mu \nu} \sigma_{i} \sigma_{j} \sigma_{\mu} \sigma_{v},
\end{aligned}
$$

where $t_{i j \mu}$ and $q_{i j \mu v}$ denote the triple-correlations and, respectively, the quadruple correlations among the respective parameters.

3) The $3^{\text {rd }}$-order moment $\mu_{3}\left(R_{k}, R_{l}, R_{m}\right)$ of three responses has the following expression [4]:

$$
\begin{aligned}
& \mu_{3}\left(R_{k}, R_{l}, R_{m}\right)=\sum_{i=1}^{N_{\alpha}} \sum_{j=1}^{N_{\alpha}} \sum_{\mu=1}^{N_{\alpha}} \frac{\partial R_{k}}{\partial \alpha_{i}} \frac{\partial R_{l}}{\partial \alpha_{j}} \frac{\partial R_{m}}{\partial \alpha_{\mu}} t_{i j \mu} \sigma_{i} \sigma_{j} \sigma_{\mu} \\
& +\frac{1}{2} \sum_{i=1}^{N_{\alpha}} \sum_{j=1}^{N_{\alpha}} \sum_{\mu=1}^{N_{\alpha}} \sum_{v=1}^{N_{\alpha}} \frac{\partial R_{k}}{\partial \alpha_{i}} \frac{\partial R_{l}}{\partial \alpha_{j}} \frac{\partial^{2} R_{m}}{\partial \alpha_{\mu} \partial \alpha_{v}}\left(q_{i j \mu \nu}-\rho_{i j} \rho_{\mu v}\right) \sigma_{i} \sigma_{j} \sigma_{\mu} \sigma_{v} \\
& +\frac{1}{2} \sum_{i=1}^{N_{\alpha}} \sum_{j=1}^{N_{\alpha}} \sum_{\mu=1}^{N_{\alpha}} \sum_{v=1}^{N_{\alpha}} \frac{\partial R_{k}}{\partial \alpha_{i}} \frac{\partial^{2} R_{l}}{\partial \alpha_{j} \partial \alpha_{\mu}} \frac{\partial R_{m}}{\partial \alpha_{v}}\left(q_{i j \mu \nu}-\rho_{i v} \rho_{j \mu}\right) \sigma_{i} \sigma_{j} \sigma_{\mu} \sigma_{v} \\
& +\frac{1}{2} \sum_{i=1}^{N_{\alpha}} \sum_{j=1}^{N_{\alpha}} \sum_{\mu=1}^{N_{\alpha}} \sum_{v=1}^{N_{\alpha}} \frac{\partial^{2} R_{k}}{\partial \alpha_{i} \partial \alpha_{j}} \frac{\partial R_{l}}{\partial \alpha_{\mu}} \frac{\partial R_{m}}{\partial \alpha_{v}}\left(q_{i j \mu \nu}-\rho_{i j} \rho_{\mu v}\right) \sigma_{i} \sigma_{j} \sigma_{\mu} \sigma_{v} .
\end{aligned}
$$

The $3^{\text {rd }}$-order sensitivities would produce terms on the right-side of Equation (3) which are higher than $4^{\text {th }}$-order in the parameters' standard deviations and are therefore not included.

The skewness, denoted as $\gamma_{1}(R)$ of a single response $R$, which indicates the degree of the distribution's asymmetry with respect to its mean, is defined as follows [4]:

$$
\gamma_{1}(R)=\frac{\mu_{3}(R)}{[\operatorname{var}(R)]^{3 / 2}} .
$$

As has been discussed in [5], correlations among the group-averaged microscopic total cross sections are not available for the PERP benchmark under consideration. When such correlations are unavailable, the maximum entropy 
principle (see, e.g., Ref. [6]) indicates that neglecting them minimizes the inadvertent introduction of spurious information into the computations of the various response moments. Also, third- and fourth-order correlations for the group-averaged microscopic total cross sections are not available in the open literature; therefore, the effects on the uncertainties they would induce in the leakage response cannot be exactly quantified. In the absence of any information whatsoever regarding the triple correlations among the group-averaged microscopic total cross sections, the most reasonable assumption (in the sense of introducing the least amount of spurious information into the system, according to the maximum entropy principle [6]) is to set $t_{i j \mu} \equiv 0$, which would be rigorously correct if the group-averaged microscopic total cross section were considered to be multivariate normally distributed quantities, even if correlated. For normally distributed parameters, the following relation also holds:

$$
q_{i j \mu v}=\rho_{i j} \rho_{\mu v}+\rho_{i \mu} \rho_{j v}+\rho_{i v} \rho_{j \mu} .
$$

When the group-averaged microscopic total cross sections are uncorrelated, the expected value of the leakage response has the following expression:

$$
[E(L)]_{t}^{(U)}=L\left(\boldsymbol{\alpha}^{0}\right)+[E(L)]_{t}^{(2, U)},
$$

where the superscript " $U$ " indicates contributions from uncorrelated parameters, the subscript $t$ indicates "total" cross section, and where the quantity $[E(L)]_{t}^{(2, U)}$ is defined as follows:

$$
[E(L)]_{t}^{(2, U)}=\frac{1}{2} \sum_{g=1}^{G} \sum_{i=1}^{I} \frac{\partial^{2} L(\boldsymbol{\alpha})}{\partial^{2} \sigma_{t, i}^{g}}\left(s_{t, i}^{g}\right)^{2}+\frac{1}{6} \sum_{g=1}^{G} \sum_{i=1}^{I} \frac{\partial^{3} L(\boldsymbol{\alpha})}{\partial^{3} \sigma_{t, i}^{g}}\left(s_{t, i}^{g}\right)^{3} t_{i i i}, G=30, I=6 \text {. (7) }
$$

In Equation (7), the quantity $s_{t, i}^{g}$ denotes the standard deviation associated with the parameter $\sigma_{t, i}^{g}$.

If the group-averaged microscopic total cross sections are uncorrelated and normally-distributed [which will be indicated by using the superscript " $(U, N)$ " in the following equations], the expected value of the leakage response does not depend on the $3^{\text {rd }}$-order sensitivities and has the following expression:

$$
[E(L)]_{t}^{(U, N)}=L\left(\boldsymbol{\alpha}^{0}\right)+[E(L)]_{t}^{(2, U, N)},
$$

where

$$
[E(L)]_{t}^{(2, U, N)} \triangleq \frac{1}{2} \sum_{g=1}^{G} \sum_{i=1}^{I} \frac{\partial^{2} L(\boldsymbol{\alpha})}{\partial \sigma_{t, i}^{g} \partial \sigma_{t, i}^{g}}\left(s_{t, i}^{g}\right)^{2}, G=30, I=6 .
$$

When the group-averaged microscopic total cross sections are uncorrelated and normally-distributed, the variance of the leakage response of the PERP benchmark takes on the following particular form:

$$
[\operatorname{var}(L)]_{t}^{(U, N)}=[\operatorname{var}(L)]_{t}^{(1, U, N)}+[\operatorname{var}(L)]_{t}^{(2, U, N)}+[\operatorname{var}(L)]_{t}^{(3, U, N)},
$$

where the first-order contribution term, $[\operatorname{var}(L)]_{t}^{(1, U, N)}$, to the variance $[\operatorname{var}(L)]_{t}^{(U, N)}$ is defined as follows, 


$$
[\operatorname{var}(L)]_{t}^{(1, U, N)} \triangleq \sum_{g=1}^{G} \sum_{i=1}^{I}\left[\frac{\partial L(\boldsymbol{\alpha})}{\partial \sigma_{t, i}^{g}}\right]^{2}\left(s_{t, i}^{g}\right)^{2}, \quad G=30, I=6 ;
$$

while the second-order contribution term, $[\operatorname{var}(L)]_{t}^{(2, U, N)}$, to the variance $[\operatorname{var}(L)]_{t}^{(U, N)}$ is defined as follows:

$$
[\operatorname{var}(L)]_{t}^{(2, U, N)} \triangleq \frac{1}{2} \sum_{g=1}^{G} \sum_{i=1}^{I}\left[\frac{\partial^{2} L(\alpha)}{\partial \sigma_{t, i}^{g} \partial \sigma_{t, i}^{g}}\left(s_{t, i}^{g}\right)^{2}\right]^{2}, \quad G=30, I=6 ;
$$

and the third-order contribution term, $[\operatorname{var}(L)]_{t}^{(3, U, N)}$, to the variance $[\operatorname{var}(L)]_{t}^{(U, N)}$ is defined as follows:

$$
[\operatorname{var}(L)]_{t}^{(3, U, N)} \triangleq \sum_{g=1}^{G} \sum_{i=1}^{I} \sum_{g^{\prime}=1}^{G} \sum_{j=1}^{I}\left[\frac{\partial L(\boldsymbol{\alpha})}{\partial \sigma_{t, i}^{g}} \frac{\partial^{3} L(\boldsymbol{\alpha})}{\partial \sigma_{t, i}^{g} \partial \sigma_{t, j}^{g^{\prime}} \partial \sigma_{t, j}^{g^{\prime}}}\right]\left(s_{t, i}^{g}\right)^{2}\left(s_{t, j}^{g^{\prime}}\right)^{2}, G=30, I=6 .
$$

For uncorrelated and normally distributed microscopic total cross sections, the third-order moment, $\left[\mu_{3}(L)\right]_{t}^{(U, N)}$, of the leakage response for the PERP benchmark takes on the following particular form:

$$
\left[\mu_{3}(L)\right]_{t}^{(U, N)}=3 \sum_{g=1}^{G} \sum_{i=1}^{I}\left[\frac{\partial L(\boldsymbol{\alpha})}{\partial \sigma_{t, i}^{g}}\right]^{2} \frac{\partial^{2} L(\boldsymbol{\alpha})}{\partial \sigma_{t, i}^{g} \partial \sigma_{t, i}^{g}}\left(s_{t, i}^{g}\right)^{4}, G=30, I=6 .
$$

Since the third-order correlations for the group-averaged microscopic total cross sections are not available, the contributions of the third-order sensitivities are neglected in Equation (14).

The skewness, $\left[\gamma_{1}(L)\right]_{t}^{(U, N)}$, of a single response $L(\boldsymbol{\alpha})$, which indicates the degree of the distribution's asymmetry with respect to its mean, is given by the following expression for uncorrelated and normally distributed parameters:

$$
\left[\gamma_{1}(L)\right]_{t}^{(U, N)}=\left[\mu_{3}(L)\right]_{t}^{(U, N)} /\left\{[\operatorname{var}(L)]_{t}^{(U, N)}\right\}^{3 / 2} .
$$

As Equation (14) indicates, if the $2^{\text {nd }}$-order sensitivities were unavailable, the third moment $\left[\mu_{3}(L)\right]_{t}^{(U, N)}$ and hence the skewness $\left[\gamma_{1}(L)\right]_{t}^{(U, N)}$ of the leakage response would vanish and the response distribution would by default be assumed to be Gaussian.

\section{Numerical Results for Third-Order Uncertainty Analysis of the PERP Leakage Response}

The effects of the first- and second-order sensitivities of the leakage response with respect to the microscopic total cross sections on the response's expected value, variance and skewness have been quantified and documented in the previous works [5] [7]. In this work, the effects of the third-order sensitivities on the response's variance and skewness are quantified by considering typical values for the standard deviations of the group-averaged microscopic total cross sections, and using these values together with the respective first-, second- and third-order sensitivities in Equations (6)-(15). The results thus obtained are presented in Table 1, considering uniform parameter standard deviations of $1 \%$ (very small), 5\% (moderate), and 10\% (large), respectively. 
Table 1. Comparison of response moments for various relative standard deviations of the uncorrelated and normally-distributed microscopic total cross sections.

\begin{tabular}{|c|c|c|c|}
\hline Relative Standard Deviation & $1 \%$ & $5 \%$ & $10 \%$ \\
\hline$L\left(\boldsymbol{\alpha}^{0}\right)$ & $1.765 \times 10^{6}$ & $1.765 \times 10^{6}$ & $1.765 \times 10^{6}$ \\
\hline$[E(L)]_{t}^{(2, U, N)}$ & $4.598 \times 10^{4}$ & $1.149 \times 10^{6}$ & $4.598 \times 10^{6}$ \\
\hline$[E(L)]_{t}^{(U, N)}=L\left(\boldsymbol{\alpha}^{0}\right)+[E(L)]_{t}^{(2, U, N)}$ & $1.811 \times 10^{6}$ & $2.914 \times 10^{6}$ & $6.363 \times 10^{6}$ \\
\hline$[\operatorname{var}(L)]_{t}^{(1, U, N)}$ & $3.419 \times 10^{10}$ & $8.549 \times 10^{11}$ & $3.419 \times 10^{12}$ \\
\hline$[\operatorname{var}(L)]_{t}^{(2, U, N)}$ & $2.879 \times 10^{9}$ & $1.799 \times 10^{12}$ & $2.879 \times 10^{13}$ \\
\hline$[\operatorname{var}(L)]_{t}^{(3, U, N)}$ & $1.308 \times 10^{10}$ & $8.713 \times 10^{12}$ & $1.308 \times 10^{14}$ \\
\hline $\begin{aligned} {[\operatorname{var}(L)]_{t}^{(U, N)}=} & {[\operatorname{var}(L)]_{t}^{(1, U, N)}+[\operatorname{var}(L)] } \\
& +[\operatorname{var}(L)]_{t}^{(3, U, N)}\end{aligned}$ & $5.015 \times 10^{10}$ & $1.083 \times 10^{13}$ & $1.630 \times 10^{14}$ \\
\hline$\left[\mu_{3}(L)\right]_{t}^{(U, N)}$ & $6.227 \times 10^{15}$ & $3.892 \times 10^{18}$ & $6.227 \times 10^{19}$ \\
\hline$\left[\gamma_{1}(L)\right]_{t}^{(U, N)}$ & 0.554 & 0.109 & 0.030 \\
\hline
\end{tabular}

As shown in Equations (9) and (14), the third-order sensitivities do not affect to the expected value $[E(L)]_{t}^{(U, N)}$ and, respectively, the third-order moment $\left[\mu_{3}(L)\right]_{t}^{(U, N)}$ when the parameters are uncorrelated and normally distributed. However, the third-order sensitivities do affect the values of the variances $[\operatorname{var}(L)]_{t}^{(3, U, N)}$ and $[\operatorname{var}(L)]_{t}^{(U, N)}$, as well as the skewness $\left[\gamma_{1}(L)\right]_{t}^{(U, N)}$, as shown in Table 1. Note that the numerical values shown in Table 1 for the firstand second-order contributions to the response moments, namely, $L\left(\alpha^{0}\right)$, $[E(L)]_{t}^{(2, U, N)}, \quad[E(L)]_{t}^{(U, N)},[\operatorname{var}(L)]_{t}^{(1, U, N)},[\operatorname{var}(L)]_{t}^{(2, U, N)}$ and $\left[\mu_{3}(L)\right]_{t}^{(U, N)}$, are the same as those presented in Table 25 from the previous work [5].

\subsection{Very Small (1\%) Relative Standard Deviations for Total Cross Sections}

When the relative standard deviations of the uncorrelated microscopic total cross sections are very small (1\%), the results presented in Table 1 show that $[E(L)]_{t}^{(2, U, N)} \approx 2.5 \% \times[E(L)]_{t}^{(U, N)}$, which indicates the effects of the $2^{\text {nd }}$-order sensitivities through $[E(L)]_{t}^{(2, U, N)}$ to the expected response value $[E(L)]_{t}^{(U, N)}$ are negligibly small. The results presented in Table 1 also indicate that $[\operatorname{var}(L)]_{t}^{(1, U, N)} \approx 68 \% \times[\operatorname{var}(L)]_{t}^{(U, N)},[\operatorname{var}(L)]_{t}^{(2, U, N)} \approx 6 \% \times[\operatorname{var}(L)]_{t}^{(U, N)}$, and $[\operatorname{var}(L)]_{t}^{(3, U, N)} \approx 26 \% \times[\operatorname{var}(L)]_{t}^{(U, N)}$, which means that the $1^{\text {st }}$-order sensitivities contribute the most (namely: 68\%) to the response variance, followed by the $3^{\text {rd }}$-order sensitivities, which contribute about $26 \%$ to the response variance, while the $2^{\text {nd }}$-order sensitivities contribute only about $6 \%$ to the response variance. Thus, for very small relative standard deviations (in this case: $1 \%$ ), the 
contributions from the $1^{\text {st }}$-order sensitivities are significantly larger than those from higher order sensitivities.

The effects arising solely from the $1^{\text {st }}-2^{\text {nd }}$ - and, respectively, $3^{\text {rd }}$-order sensitivities of the leakage response on the standard deviations for the PERP leakage response on the uncertainties, are also compared to one another in Figure 1. Assuming that all the microscopic total cross sections are uncorrelated and have uniform relative standard deviations of $1 \%$, Figure 1 presents results for the following quantities: 1 ) the PERP leakage response $L\left(\alpha^{0}\right) ; 2$ ) the expected value $[E(L)]_{t}^{(U, N)}$ of the PERP leakage response; 3$)$ the standard deviation, $S D^{(1)}$, for the PERP leakage response arising solely from the $1^{\text {st }}$-order sensitivities (in green); 4) the standard deviation, $S D^{(2)}$, for the PERP leakage response arising solely from the $2^{\text {nd }}$-order sensitivities; and 5) the standard deviations, $S D^{(3)}$, for the PERP leakage response stemming solely from the $3^{\text {rd }}$-order sensitivities. In Figure 1, the green-colored plots involve solely $1^{\text {st }}$-order sensitivities, while the red-colored plots depict the contributions from $2^{\text {nd }}$ - and $3^{\text {rd }}$-order sensitivities. The results presented in Figure 1 depict visually that $S D^{(1)} \gg S D^{(3)} \gg S D^{(2)}$, as established by the numerical results presented in Table 1.

As indicated in Table 1, all of the 1st-, 2nd- and 3rd-order sensitivities give rise to a positive response skewness, thus causing the response distribution to be asymmetrically "skewed" towards the "positive direction" from the response's expected value $[E(L)]_{t}^{(U, N)}$. When only the $1^{\text {st }}$ - and $2^{\text {nd }}$-order sensitivities are considered, it has been shown in [5] that $\left[\gamma_{1}(L)\right]_{t}^{(U, N)}=0.872$. Including the contributions from the $3^{\text {rd }}$-order sensitivities yields $\left[\gamma_{1}(L)\right]_{t}^{(U, N)}=0.554$, indicating that the distribution of the leakage response in the space of parameters is more symmetrical in reality.

\subsection{Typical (5\%) Relative Standard Deviations for Total Cross Sections}

Relative standard deviations between $5 \%$ to $10 \%$ are typical for the microscopic total cross sections for most of the nuclides tabulated in nuclear data banks. The third column of Table 1 presents results when considering a uniform relative standard deviation of $5 \%$ for the uncorrelated microscopic total cross sections of the isotopes included in the PERP benchmark. These results show that $[E(L)]_{t}^{(2, U, N)} \approx 39 \% \times[E(L)]_{t}^{(U, N)}$, indicating the contributions from the $2^{\text {nd }}$-order sensitivities to the expected response value have become comparable, for $5 \%$ relative standard deviations, to the computed leakage value $L\left(\alpha^{0}\right)$, thus causing a significant shift, by about $40 \%$, of the expected value for the leakage response $[E(L)]_{t}^{(U, N)}$ by comparison to the computed value $L\left(\boldsymbol{\alpha}^{0}\right)$. As has been discussed in [5], the customary procedure of neglecting second (and higher) order sensitivities and considering that the computed value, $L\left(\boldsymbol{\alpha}^{0}\right)$, is the actual expected (i.e., mean) value of the distribution, would be about $40 \%$ in error for $5 \%$ relative standard deviations for uncorrelated total cross sections. 


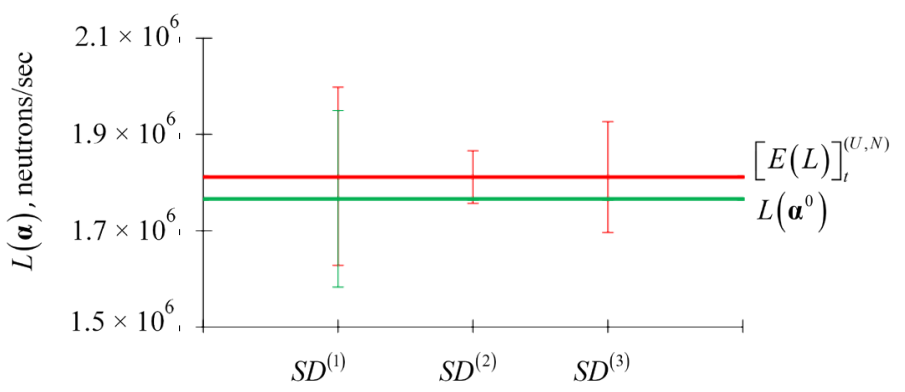

Figure 1. Comparison of $L\left(\alpha^{0}\right) \pm S D^{(1)}$ (in green), $[E(L)]_{t}^{(U, N)} \pm S D^{(1)}, S D^{(2)}, S D^{(3)}$ (in red), due to $1 \%$ standard deviations of the uncorrelated microscopic total cross sections.

The results presented in the third column of Table 1 also indicate that $[\operatorname{var}(L)]_{t}^{(1, U, N)} \approx 8 \% \times[\operatorname{var}(L)]_{t}^{(U, N)},[\operatorname{var}(L)]_{t}^{(2, U, N)} \approx 17 \% \times[\operatorname{var}(L)]_{t}^{(U, N)}$ and $[\operatorname{var}(L)]_{t}^{(3, U, N)} \approx 75 \% \times[\operatorname{var}(L)]_{t}^{(U, N)}$, which means that the first-, second- and third-order sensitivities contribute about $8 \%, 17 \%$ and $75 \%$, respectively, to the response variance $[\operatorname{var}(L)]_{t}^{(U, N)}$. The effects of the $1^{\text {st }}-2^{\text {nd }}-$ and $3^{\text {rd }}$-order sensitivities on the quantities $L\left(\alpha^{0}\right) \pm S D^{(1)}$ and $[E(L)]_{t}^{(U, N)} \pm S D^{(1)}, S D^{(2)}, S D^{(3)}$, in conjunction with $5 \%$ standard deviations for the uncorrelated microscopic total cross sections, are illustrated in Figure 2. As illustrated in the figure, $S D^{(3)} \gg S D^{(2)}>S D^{(1)}$, which indicates that the contribution from the $2^{\text {nd }}$-order sensitivities have exceeded the contributions from the firs-order ones. As in the previously studied case of " $1 \%$ uniform standard deviations," the contributions from the third-order sensitivities remain dominant, being much larger than the contributions from either the $1^{\text {st }}$ - or the $2^{\text {nd }}$-order sensitivities. Hence, neglecting the third-order sensitivities would cause a significant error in quantifying the standard deviation of the leakage response.

The skewness $\left[\gamma_{1}(L)\right]_{t}^{(U, N)}=0.109$ remains positive for the " $5 \%$ case" when the contributions of the $3^{\text {rd }}$-order sensitivities are included, but is smaller than the skewness obtained for the " $1 \%$ case." This comparison indicates that larger standard deviations for the uncorrelated microscopic total cross sections tend to reduce the skewness and therefore render the distribution of the leakage response more symmetrical about the mean value $[E(L)]_{t}^{(U, N)}$ in the parameter space. Notably, if the $3^{\text {rd }}$-order sensitivities were neglected when considering a uniform $5 \%$ relative standard deviation for the uncorrelated microscopic total cross sections, the skewness would take on the value $\left[\gamma_{1}(L)\right]_{t}^{(U, N)}=0.899$, as shown in [5], which would be ca. 900\% larger (erroneously) than the more accurate result obtained by including the effects of the $3^{\text {rd }}$-order sensitivities.

\subsection{Large (10\%) Relative Standard Deviations for Total Cross Sections}

The results obtained by considering a $10 \%$ uniform relative standard deviation for each of the uncorrelated microscopic total cross sections are presented in the 


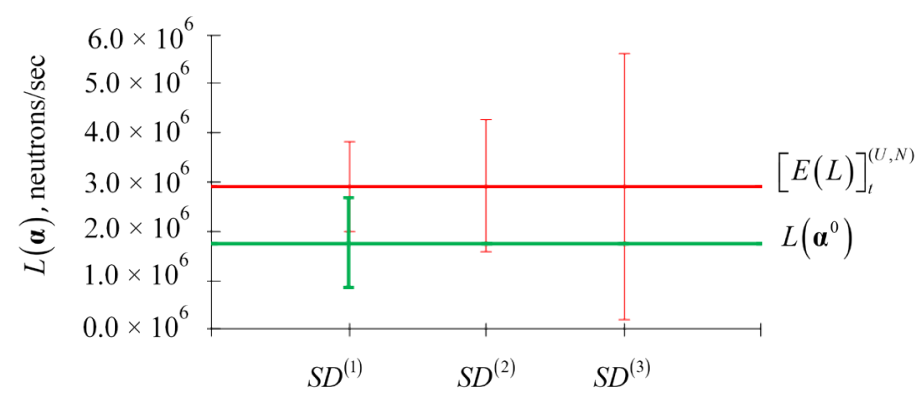

Figure 2. Comparison of $L\left(\alpha^{0}\right) \pm S D^{(1)}$ (in green), $[E(L)]_{t}^{(U, N)} \pm S D^{(1)}, S D^{(2)}, S D^{(3)}$ (in red), due to $5 \%$ standard deviations of the uncorrelated microscopic total cross sections.

last column of Table 1. These results display the same trends as discussed in the previous subsection for the " $5 \%$ case", except that the respective trends are significantly amplified by comparison to the corresponding results for the " $5 \%$ case". For the " $10 \%$ case", the following results are observed:

$[\operatorname{var}(L)]_{t}^{(1, U, N)} \approx 2 \% \times[\operatorname{var}(L)]_{t}^{(U, N)},[\operatorname{var}(L)]_{t}^{(2, U, N)} \approx 18 \% \times[\operatorname{var}(L)]_{t}^{(U, N)}$ and $[\operatorname{var}(L)]_{t}^{(3, U, N)} \approx 80 \% \times[\operatorname{var}(L)]_{t}^{(U, N)}$, which means that the contributions stemming from the third-order sensitivities to the response variance $[\operatorname{var}(L)]_{t}^{(U, N)}$ are remarkably larger than the contributions stemming from the corresponding first- and second-order sensitivities.

The $10 \%$ relative standard deviations in the parameters in conjunction with the $2^{\text {nd }}$-order sensitivities also cause a larger deviation of the leakage response's expected value from its computed value: as shown in the last column of Table 1: $[E(L)]_{t}^{(2, U, N)} \approx 260 \% \times L\left(\alpha^{0}\right) \approx 72 \% \times[E(L)]_{t}^{(U, N)}$; this result indicates that the second-order term is about 2.6 times larger than the computed leakage value $L\left(\boldsymbol{\alpha}^{0}\right)$, contributing $72 \%$ of the expected value $[E(L)]_{t}^{(U, N)}$ of the leakage response.

For the " $10 \%$ case", the effects of the $1^{\text {st }}-2^{\text {nd }}$ - and $3^{\text {rd }}$-order sensitivities on the uncertainties of the leakage response and the expected value, i.e., $L\left(\alpha^{0}\right) \pm S D^{(1)}$ and $[E(L)]_{t}^{(U, N)} \pm S D^{(1)}, S D^{(2)}, S D^{(3)}$, are displayed in Figure 3, which clearly indicates that $S D^{(3)} \gg S D^{(2)}>S D^{(1)}$. It is also important to note that the negative results displayed in the green-colored plots for $L\left(\alpha^{0}\right)-S D^{(1)}$ and in the red colored plots for $[E(L)]_{t}^{(U, N)}-S D^{(3)}$ in Figure 3, indicate that if the uncorrelated parameters are affected by relative standard deviations of $10 \%$, the $3^{\text {rd }}$-order Taylor series expansion of the response around the nominal parameter values (which underlies the mathematical expressions for computing response uncertainties that stem from parameter uncertainties) is an inadequate representation of the response distribution. An investigation of the consequences of this observation is beyond the scope of this work but is currently underway.

As shown in Table 1, the skewness for the " $10 \%$ case" is smaller than the skewness for the corresponding " $1 \%$ case" and/or " $5 \%$ case". Furthermore, it has been shown (for $10 \%$ standard deviations in uncorrelated microscopic total cross 


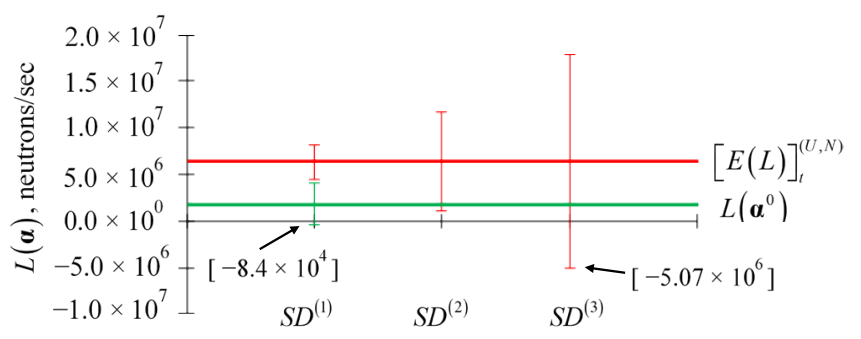

Figure 3. Comparison of $L\left(\alpha^{0}\right) \pm S D^{(1)}$ (in green), $[E(L)]_{t}^{(U, N)} \pm S D^{(1)}, S D^{(2)}, S D^{(3)}$ (in red), due to $10 \%$ standard deviations of the uncorrelated microscopic total cross sections.

sections) in [5] that if only the contributions stemming from the $1^{\text {st }}$ and $2^{\text {nd }}$-order sensitivities are taken into account, the corresponding skewness is much larger than the skewness obtained when the contributions from the $3^{\text {rd }}$-order sensitivities are also added in, namely: 1) $\left[\gamma_{1}(L)\right]_{t}^{(U, N)}=0.341$ without the $3^{\text {rd }}$-order sensitivities, but 2) $\left[\gamma_{1}(L)\right]_{t}^{(U, N)}=0.030$ when all of the contributions stemming from the $1^{\text {st }}$ - $2^{\text {nd }}$ - and $3^{\text {rd }}$-order sensitivities are accounted for. This results again indicates that the distribution of the leakage response in the parameter space becomes more symmetrical about its expected value when the contributions from 3rd-order sensitivities are accounted for.

It is important to emphasize that the results presented in Table 1 for $[\operatorname{var}(L)]_{t}^{(3, U, N)}$ considered the contributions from only 96,840 uncorrelated elements out of the total of the total $180^{3}=5832000$ elements of the matrix of $3^{\text {rd }}$-order sensitivities $\boldsymbol{S}^{(3)}\left(\sigma_{t, j}^{g}, \sigma_{t, k}^{g^{\prime}}, \sigma_{t, l}^{g^{\prime \prime}}\right), j, k, l=1, \cdots, 6 ; g, g^{\prime}, g^{\prime \prime}=1, \cdots, 30$. On the other hand, the results presented in [2] indicate that some of the excluded mixed $3^{\text {rd }}$-order relative sensitivities of the leakage response with respect to the microscopic total cross sections have large values. However, the effect of these large mixed third-order sensitivities on the uncertainties in the response distribution cannot be considered since the corresponding correlations among the microscopic total cross sections are not available.

\section{Conclusions}

This work has quantified the contributions of the $(180)^{3}$ third-order mixed sensitivities, $\partial^{3} L / \partial \sigma_{t, j}^{g} \partial \sigma_{t, k}^{g^{\prime}} \partial \sigma_{t, l}^{g^{\prime \prime}}, j, k, l=1, \cdots, 6 ; g, g^{\prime}, g^{\prime \prime}=1, \cdots, 30$ of the PERP benchmark's total leakage response with respect to the benchmark's 180 microscopic total cross sections, to the benchmark's leakage response moments (expected values, variance and skewness), and compared these contributions with those stemming from the corresponding first- and second-order sensitivities. The following conclusions can be drawn from the results reported in this work:

1) The results shown in Table 1 indicate that the importance of the $3^{\text {rd }}$-order sensitivities increases with increasing uncertainties in the PERP benchmark's microscopic total cross sections.

2) The contributions of the $3^{\text {rd }}$-order sensitivities to the response's variance surpass the contributions of the $1^{\text {st }}$-order and $2^{\text {nd }}$-order sensitivities to the re- 
sponse's variance already for relatively small (ca. 5\%) standard deviations for the uncertain cross sections. These effects are rapidly amplified as the uncertainties in the cross sections increase. In particular, for a uniform standard deviation of $10 \%$, the $3^{\text {rd }}$-order sensitivities contribute ca. $80 \%$ of the response variance $[\operatorname{var}(L)]_{t}^{(U, N)}$. By comparison, the first-order sensitivities contribute ca. $2 \%$, while the second-order sensitivities contribute ca. $18 \%$ of the response variance $[\operatorname{var}(L)]_{t}^{(U, N)}$. Thus, neglecting the contributions stemming from the third-order sensitivities would cause a large non-conservative error by under-reporting the response variance by a factor of $506 \%$.

3) The $1^{\text {st }}-, 2^{\text {nd }}$ - and $3^{\text {rd }}$-order sensitivities all yield a positive response skewness, indicating that the response distribution is asymmetrically "skewed" towards values higher than the response's expected value $[E(L)]_{t}^{(U, N)}$. When the relative standard deviations of the microscopic total cross sections are increased, the magnitude of the response skewness remains positive but decreases, indicating that the response distribution becomes more symmetrical around the response's expected value.

4) The effects of the $3^{\text {rd }}$-order sensitivities are towards reducing the response skewness, thereby reducing the asymmetry of the leakage response distribution in the space of parameters (total cross sections); this effect is the more pronounced the larger the parameter's standard deviations. In particular, for a uniform relative standard deviation of $10 \%$ for the uncorrelated microscopic total cross sections, the skewness is $\left[\gamma_{1}(L)\right]_{t}^{(U, N)}=0.341$ when only the $1^{\text {st }}$ - and $2^{\text {nd }}$-order sensitivities are considered; however, when the $3^{\text {rd }}$-order sensitivities are also included, the magnitude of the skewness decreases to $\left[\gamma_{1}(L)\right]_{t}^{(U, N)}=0.030$.

5) Since the actual correlations between the microscopic total cross sections for the PERP benchmark are unavailable, the effects of the mixed $3^{\text {rd }}$-order sensitivities on the response moments (e.g., expected value, variance and skewness) are underestimated; the same is true of the effects stemming from the $1^{\text {st }}$ - and $2^{\text {nd }}$-order sensitivities. Therefore, it would be very important for future experimental research to obtain values for the correlations that might exist among the various cross sections. Correlations among the total cross sections could provide significantly larger contributions to the response moments in addition to those stemming from the parameters' standard deviations.

6) Relative standard deviations of $10 \%$ in the total cross sections can produce unphysical values for the standard deviation of the response distribution, as displayed in Figure 3, which indicates that the $3^{\text {rd }}$-order Taylor series expansion of the response around the nominal parameter values (which underlies the mathematical expressions for computing response uncertainties that stem from parameter uncertainties) is an inadequate representation of the response distribution. An investigation of the consequences of this observation is currently underway, commencing with the examination of the convergence properties of the series of higher-order sensitivities. 


\section{Conflicts of Interest}

The authors declare no conflicts of interest regarding the publication of this paper.

\section{References}

[1] Cacuci, D.G. and Fang, R. (2020) Third Order Adjoint Sensitivity and Uncertainty Analysis of an OECD/NEA Reactor Physics Benchmark: I. Mathematical Framework. American Journal of Computational Mathematics, 10, 503-528.

https://doi.org/10.4236/ajcm.2020.104029

[2] Fang, R. and Cacuci, D.G. (2020) Third Order Adjoint Sensitivity and Uncertainty Analysis of an OECD/NEA Reactor Physics Benchmark: II. Computed Sensitivities. American Journal of Computational Mathematics, 10, 529-558. https://doi.org/10.4236/ajcm.2020.104030

[3] Valentine, T.E. (2006) Polyethylene-Reflected Plutonium Metal Sphere Subcritical Noise Measurements, SUB-PU-METMIXED-001. In: International Handbook of Evaluated Criticality Safety Benchmark Experiments, NEA/NSC/DOC(95)03/I-IX; Organization for Economic Co-Operation and Development, Nuclear Energy Agency, Paris, France.

[4] Cacuci, D.G. (2020) Third Order Adjoint Sensitivity Analysis of Reaction Rate Responses in a Multiplying Nuclear System with Source. Annals of Nuclear Energy, 151, 107924. https://doi.org/10.1016/j.anucene.2020.107924

[5] Cacuci, D.G., Fang, R. and Favorite, J.A. (2019) Comprehensive Second-Order Adjoint Sensitivity Analysis Methodology ( $\left.2^{\text {nd }}-A S A M\right)$ Applied to a Subcritical Experimental Reactor Physics Benchmark: I. Effects of Imprecisely Known Microscopic Total and Capture Cross Sections. Energies, 12, 4219. https://doi.org/10.3390/en12214219

[6] Cacuci, D.G. (2018) BERRU Predictive Modeling: Best Estimate Results with Reduced Uncertainties. Springer, Heidelberg, Germany, New York, NY, USA. https://doi.org/10.1007/978-3-662-58395-1

[7] Fang, R. and Cacuci, D.G. (2020) Comprehensive Second-Order Adjoint Sensitivity Analysis Methodology (2nd-ASAM) Applied to a Subcritical Experimental Reactor Physics Benchmark: VI. Overall Impact of 1st- and 2nd-Order Sensitivities on Response Uncertainties. Energies, 13, 1674. https://doi.org/10.3390/en13071674 